Юргинский технологический институт. - Томск: Изд-во Томского политехнического университета. - 2014.

3. Сапрыкин А.С. Математические методы в горном деле. / IV Всероссийская научно-практическая конференция с элементами научной школы для студентов и учащейся молодежи «Прогрессивные технологии и экономика в машиностроении»/ Юргинский технологический институт. - Томск: Изд-во Томского политехнического университета. - 2013.

4. Ермуратский П.В. Электротехника и электроника / П.В. Ермуратский, Г.П. Лычкина, Ю.Б. Минкин. - М.: ДМК Пресс, 2013. - 416 с.

5. Симоненко О.Д. Из истории развития технических наук //http://www.portalslovo.ru/impressionism/36325.php.

6. Афонин В.В., Акулинин И.Н., Ткаченко А.А. Сборник задач по электротехнике: Учеб. пособие. В 3-х ч. Тамбов: Изд-во Тамб. гос. техн. ун-та, 2004. Ч. $1.80 \mathrm{c}$.

7. Комплексные числа в электротехнике [электронный ресурс]. Режим доступа: //http://sibac.info/node/34462//.

\title{
Принципы и методы параллельного программирования
}

\author{
Павлюкевич C.A., студент, \\ Северо-Восточный федеральный университет, \\ 2. Якутск \\ E-mail: necro0809@gmail.com
}

Научный руководитель: к.п.н., доцент Протодьяконова Г.Ю.

В данной работе рассмотрены особенности параллельного программирования. Выделены основные подходы к параллельному программированию, определена его значимость и отмечены отрицательные аспекты.

В век высоких технологий мощность современных персональных компьютеров значительно возросла. Сейчас самые дешёвые процессоры содержат в себе от двух и более ядер. Для эффективного использования их вычислительной мощности применяются методы параллельного программирования. Например, организация работы сложных алгоритмов наиболее эффективна при использовании нескольких процессоров в сравнении с одним при помощи последовательных методов.

Понятие параллелизма может показаться на первый взгляд очень сложным и пугающим. На самом деле это не так. Если проводить параллели с реальном миром, то способы организации параллелизма можно представить так. Допустим, есть некая школа, которая владеет собственными ресурсами: классы, учебные пособия и так далее. Это будет наша операционная система. Каждый преподаватель даёт уроки по каким-то предметам, в отдельных кабинетах и в определённое время. Иными словами, преподаватель - это поток, который использует определённые ресурсы - время, а кабинет - это процесс.

Может быть ещё один вариант. В одном кабинете решили совместить несколько занятий. Каждый преподаватель своей части аудитории читает лекции. Данный способ 
сопряжён с некоторыми трудностями. Его эффективность, по сравнению с первым способом, гораздо ниже по причине множества отвлекающий факторов. Возвращаясь обратно от наглядных примеров к параллельному программированию, можно сделать вывод о том, что при использовании первого способа, разделения задач по процессам, обеспечивается высокая безопасность данных процесса, так как адресное пространство каждого из процессов независимо друг от друга. Однако данный способ сопряжён с рядом трудностей - для каждого процесса выделяется своя часть ресурсов; необходимо обеспечивать взаимодействие между адресными пространствами разных процессов. Операция выделения ресурсов для каждого из процессов достаточно трудоёмка. В тоже время второй способ, разделение задач по потокам, гораздо проще организовать. Ресурсы системы выделяются на один процесс и делятся между потокам внутри этого процесса. Так как адресное пространство между потоками общее, то не возникает сложностей при организации взаимодействия между потоками. Однако у данного способа есть один существенных недостаток - низкая безопасность и целостность данных. Один поток может по неосторожности программиста повредить данные другого потока, что в итоге скажется на результат работы программы.

На основании исследования выявлено, что использование параллелизма позволяет значительно увеличить производительность выполнения задач и алгоритмов, за счёт разбиения задач на отдельные части для их параллельного выполнения. Примером использования параллелизма может являться работа элементарного видеопроигрывателя. Без использования параллелизма комфортная работа с приложением была бы затруднена - приложению приходилось бы постоянно отвлекаться от воспроизведения файла для проверки того, какие действия совершил пользователь с графическим интерфейсом приложения. Данный способ параллелизма называется параллелизмом задач. В тоже время, параллелизм может применяться и для обработки огромного массива данных. Например, выполнение одновременной конвертации файлов из одного формата в другой. Данный подход называет параллелизмом по данным - когда одновременно выполняются множество одних и тех же задач.

Таким образом, мы пришли к выводу, что существует два основных подхода к организации параллелизма - за счёт нескольких процессов и за счёт нескольких потоков. Каждый из способов имеет ряд своих преимуществ и недостатков. За счёт низких расходов ресурсов и легкости организации считается способ разбиения на потоки считается самым популярным[1]. Однако использовании параллелизма требует от программиста взвешенного решения. Часто получается так, что параллельное программирование задачи или задачи не выгодно, а расходы превышают полученную выгоду.

Для применения параллелизма существует две основные причины: разделение обязанностей и увеличение производительности.

Разделение обязанностей является приоритетным направлением в программировании. Группировка взаимосвязанных и разделение не связных частей кода позволяет добиться «чистого» кода - структура программы становится проще для понимания, упрощается тестирование, дальнейшее сопровождение и, соответственно, уменьшается количество ошибок, а также уменьшаются риск несения ненужных финансовых затрат на рефакторинг программного кода.

В качестве примера разделения обязанностей рассмотрим элементарную программу-будильник. Будильник использует множество объектов-таймеров, которые 
указывают операционной системе в какой момент времени необходимо что-то сделать. Пользователь может установить сразу несколько таймеров одновременно на разные даты. Программа должна не только обеспечивать взаимодействие пользователя с её интерфейсом, но и ожидать сообщения от таймеров. И всё это необходимо делать одновременно. Если бы программа была написана без использования приёмов параллельного программирования, то, в случае с будильником, интерфейс программы перестал бы отвечать после первой установки объекта-таймера в занятое состояние. Или, в лучше случае, интерфейс отвечал бы пользователю, но с некоторой задержкой программе требовалось бы периодически проверять, какие действия совершил пользователя с интерфейсом, не сработал ли таймер и так далее. Всё это, конечно же, крайне неудобно. При использовании приёмов параллельного программирования всех вышеперечисленных неудобств можно избежать.

Стоить отметить, что в данном случае количество потоков не зависит от количества ядер, так как назначение этого способа сугубо для чистоты дизайна, а не для увеличения производительности.

Для увеличения производительности используют два способа параллельного программирования: распараллеливание по задачами и распараллеливание по данными. Распараллеливание по задачам предполагает разбиение задачи на части и выполнения их параллельно, тем самым, сократив общее время выполнения. Иными словами - один поток выполняет одну часть обработку, другой другую. Данный способ не смотря на кажущуюся простоту реализации на деле таковым не является. Это сопряжено с наличием зависимости между разными частями программы. Распараллеливание по данным означает, что каждый поток выполняет одну и ту же операцию в один момент времени.

Алгоритмы, которые легко поддаются распараллеливанию, называются естественно параллельными. Они с легкостью подвергаются масштабированию, то есть если число имеющихся аппаратных потоков увеличивается, то и степень параллелизма алгоритма возрастает [1]. Примером первого способа может служить простейший пример вычисления выражения $\mathrm{a} * \mathrm{~b}+\mathrm{b} * \mathrm{c}$. Здесь задачу можно поделить на два потока в одном производим вычисление a * b, во втором - b * c. Конечно, особого повышения производительности пользователь не заметит, но если таких операций, скажем, более миллиона, и сложность вычисления больше, чем простое перемножение двух операндов, то в этом случае можно говорить о значительном, или хотя бы заметном, повышении производительности.

Примером работы второго способа может являться обработка большого массива данных - декодирование множества видео файлов с помощью одного алгоритма в один момент времени.

Стоит сделать важное замечание, что не всегда эти способы приводят к увеличению производительности. Иногда, они помогают открывать новые возможности. Так, если разные области изображения можно обрабатывать одновременно, то впоследствии, можно обрабатывать видео более высокого разрешения[1].

Помимо понимания выгоды, которую может привести использование приёмов параллельного программирования, не маловажно понимать и все отрицательные аспекты этой технологии. Существует единственная причина не использовать параллелизм - когда затраты превосходят выгоду. В большинстве случаев, использование параллелизма при проектировании программного обеспечения 
значительно усложняет код программы для понимания. Если затраты от использования параллелизма превышают его выгоду, то стоит вовсе отказаться. Выгода от использования параллелизма может быть меньше ожидаемого или отсутствовать вовсе. Операционной системы требуется время на выделение ядра и памяти для стека, сообщение информации планировщику и так далее. В данном примере вычисления займут меньше времени, чем подготовительные операции для организации параллелизма. Поэтому лучшим проектным решением будет произведение вычисление при помощи последовательного программирования без параллелизма.

Для исследования эффекта от применения параллелизма разберём рекурсивный алгоритм нахождения числа Фибоначчи. Данный алгоритм можно представить в виде дерева (рисунок 1). Стоит отметить, что сложность рекурсивного алгоритма равна $\theta=$ $2^{n}$. Это делает алгоритм достаточно затратным по времени. Для нахождения 55 числа Фибоначчи при использовании метода последовательного программирования понадобится в зависимости от мощности процессора примерно 5591 секунд (около 2 часов).

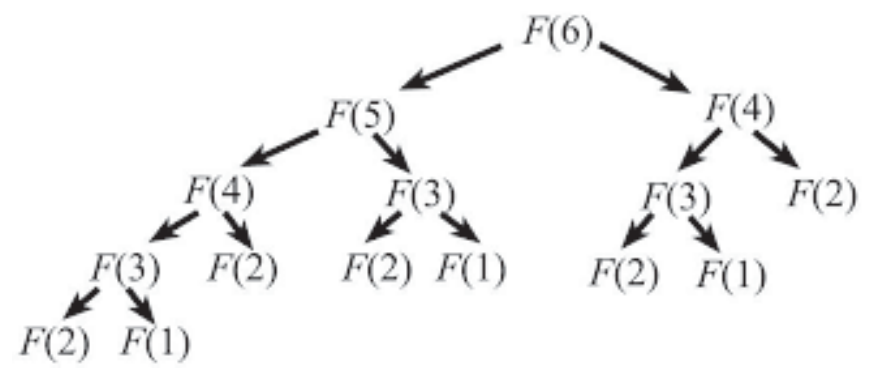

Рис. 3. Представление алгоритма в виде дерева для 6 числа

При проектировании алгоритма применялись методы последовательного и параллельного программирования. Используемые способы параллелизации:

- разделение количества итерации на группы - каждую группу обрабатывает своё ядро;

- распределение каждой итерации на ядро (управляемое планирование).

Результаты вычисления представлены на рисунке 2.

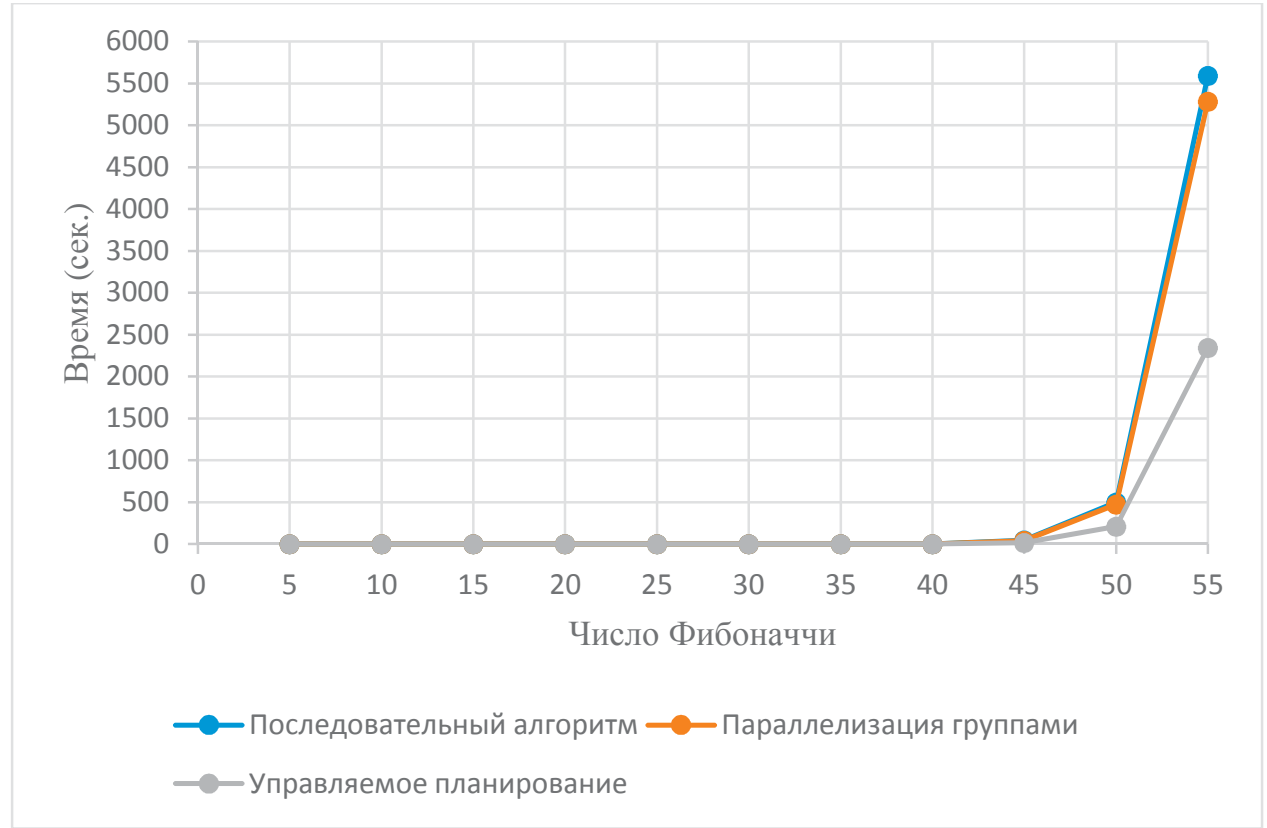

Рис. 2. Время выполнение алгоритма при разных подходах 
Анализируя полученные результаты, можно сделать вывод, что последовательный способ является самым не эффективным - время нахождения 55 числа составляет примерно 5591 секунд (около 2 часов). В то же время, параллелизация группами не даёт существенного прироста в производительности - 5283 секунд. Это связанно с необходимости ожидания завершения работы потока. При достижении границы группы осуществляется блокировка потоков до завершения работы последнего (барьерная синхронизация). Наиболее эффективным из используемых способов оказалось управляемое планирование: время нахождения 55 числа составило 2346 секунд (около 39 минут), что примерно в 2.3 раза эффективней последовательного алгоритма.

На основании проведённого исследования, можно сделать вывод, что использование приёмов параллельного программирования в наш век является достаточно эффективным способом увеличения производительности приложения. Однако стоит заметить, что выбор того или иного способа организации параллелизма, как и в целом использование методов параллельного программирования, требует взвешенного решения. В некоторых случаях параллелизм может отрицательно сказаться на производительности.

\section{Список литературы:}

1. Энтони Уильямс. Параллельное программирование на $\mathrm{C}++$ в действии. Практика разработки многопоточных программ. ДМК Пресс, 2016, 672 с.

2. Параллельные заметки №3 - базовые конструкции OpenMP // https://habrahabr.ru/company/intel/blog/85273/

3. Введение в OpenMP: параллельное программирование на $\mathrm{C}++$ // https://software.intel.com/ru-ru/blogs/2011/11/21/openmp-c

\section{Разработка информационной системы «Расписание занятий»}

Переверзева К.А., студентка, Технический институт (филиал) Северо-Восточного федерального университета, 2. Нерюнгри E-mail: pereverzeva.k@bk.ru

Научный руководитель: к.м.н., доцент Похорукова М.Ю.

В настоящее время, несмотря на повышение компьютеризации общества, в сфере образования до сих пор нет средств, позволяющих в достаточной мере автоматизировать процесс ведения документации и отчетности [1]. Большую часть своего времени преподаватели тратят на оформление различной документации и отчетов, поэтому в многих общеобразовательных учреждениях используются электронные системы, облегчая тем самым работу сотрудников. Так, например, процесс составления расписания, а также его корректировки при необходимости, удобнее всего осуществлять с помощью современных информационных технологий.

В данной статье рассматривается разработка информационной системы «Расписание занятий», которая должна содержать информацию о группах, 